\title{
Short communication: Sensitivity of estrus alerts and relationship with timing of the luteinizing hormone surge
}

\author{
Ines Adriaens, ${ }^{1 *}$ Wouter Saeys, ${ }^{1}$ Chris Lamberigts, ${ }^{2}$ Mario Berth, ${ }^{3}$ Katleen Geerinckx, ${ }^{4}$ Jo Leroy, ${ }^{5}$ \\ Bart De Ketelaere, ${ }^{1}$ and Ben Aernouts ${ }^{1,6}$ \\ ${ }^{1}$ Department of Biosystems, MeBioS, KU Leuven, Kasteelpark Arenberg 30, Box 2456, 3001, Heverlee, Belgium \\ 2Department of Biosystems, Livestock Physiology, KU Leuven, Kasteelpark Arenberg 30, Box 2456, 3001, Heverlee, Belgium \\ ${ }^{3}$ Department of Immunology, Algemeen Medisch Laboratorium, E. Vloorsstraat 9, 2020 Antwerp, Belgium \\ ${ }^{4}$ Hooibeekhoeve, Province of Antwerp, Hooibeeksedijk 1, 2440 Geel, Belgium \\ ${ }^{5}$ Faculty of Pharmaceutical, Biomedical and Veterinary Sciences, University of Antwerp, Universiteitsplein 1, Wilrijk, Belgium \\ ${ }^{6}$ Department of Microbial and Molecular Systems, Cluster for Bioengineering Technology, KU Leuven, Campus Geel, 2440 Geel, Belgium
}

\section{ABSTRACT}

Both the sensitivity of an estrus detection system and the consistency of alarms relative to ovulation determine its value for a farmer. The objective of this study was to compare an activity-based system and a milk progesterone-based system for their ability to detect estrus reliably, and to investigate how their alerts are linked to the time of the LH surge preceding ovulation. The study was conducted on an experimental research farm in Flanders, Belgium. The activity alerts were generated by a commercial activity meter (ActoFIT, DeLaval, Tumba, Sweden), and milk progesterone was measured using a commercial ELISA kit. Sensitivity and positive predictive value of both systems were calculated based on 35 estrus periods over $43 \mathrm{~d}$. Blood samples were taken for determination of the LH surge, and the intervals between timing of the alerts and the LH surge were investigated based on their range and standard deviation (SD). Activity alerts had a sensitivity of $80 \%$ and a positive predictive value of $65.9 \%$. Alerts were detected from $39 \mathrm{~h}$ before until $8 \mathrm{~h}$ after the LH surge (range: $47 \mathrm{~h}, \mathrm{SD}: 16 \mathrm{~h}$ ). Alerts based on milk progesterone were obtained from a recently developed monitoring algorithm using a mathematical model and synergistic control. All estruses were correctly identified by this algorithm, and the LH surge followed, on average, $62 \mathrm{~h}$ later. Using the mathematical model, model-based indicators for the estimation of ovulation time can be calculated. Depending on which modelbased indicator was used, ranges of 33 to $35 \mathrm{~h}$ and $\mathrm{SD}$ of about $11 \mathrm{~h}$ were obtained. Because detection of the LH surge was very labor intensive, only a limited number of potential estrus periods could be studied.

Received August 8, 2018.

Accepted October 8, 2018.

*Corresponding author: ines.adriaens@kuleuven.be
Key words: estrus detection, alerts, luteinizing hormone (LH) surge, sensitivity

\section{Short Communication}

Both maximal detection of estrus and timely insemination are important to improve on-farm fertility performance (Hockey et al., 2010a). Although the latter is strictly associated with ovulation time, the link between ovulation time and estrus alerts provided by automated estrus detection systems is seldom researched due to the high workload and costs of these studies (Roelofs, 2005). In this study, we examined the sensitivity and positive predictive value (PPV) of an activity-based system and a progesterone (P4)-based system (periods $1+2)$; subsequently, we investigated their links to the LH surge preceding ovulation (period 2).

The study was approved by the ethical committee of KU Leuven (ID P010/2017) and conducted on an experimental dairy farm in Flanders, Belgium. Twentytwo regularly cycling Holstein-Friesian cows, aged 3.4 $\pm 1.2 \mathrm{yr}$ (mean $\pm \mathrm{SD}$ ) with parities 1 to 4 and 30 to 293 DIM at the start of the study were included. The cows were milked with an automated milking system (VMS, DeLaval, Tumba, Sweden) and fed a mixed ration of grass and corn silage and supplemented with concentrates. Over $53 \mathrm{~d}$, a mixed milk sample of each milking was automatically collected by the sampling unit (VMX, DeLaval), following the procedure of the DHI protocol prescribed by ICAR (2014), and stored at $-20^{\circ} \mathrm{C}$. At the end of the trial, the certified laboratory MCC-Vlaanderen (Lier, Belgium) determined the P4 concentration using an ELISA kit (Ridgeway, Gloucester, UK), the details of which are described in Adriaens et al. (2017). The P4 concentration of each sample was also measured automatically on-farm by the Herd Navigator system (Lattec, DeLaval, Hillerød, Denmark). This device allowed estimation of the tim- 
ing of luteolysis without the need to synchronize the ovaries. Based on these data, a reference estrus period (REP) was defined as a period of low milk P4 ( $<5 \mathrm{ng} /$ $\mathrm{mL}$ ) of minimum 5 and maximum $10 \mathrm{~d}$. During the period from 15 to $50 \mathrm{~h}$ after the start of the REP, a preovulatory follicle $\geq 13 \mathrm{~mm}$ was detected by an experienced veterinarian using a transrectal ultrasound scanner (A6v, Sonoscape Medical Corp., Shenzhen, China; Hockey et al., 2010a,b). Disappearance of this follicle and formation of a corpus luteum was confirmed by transrectal ultrasound within 7 to $10 \mathrm{~d}$ after the start of the REP. This methodology allowed us to identify all estruses in the study cows, including silent estruses, while avoiding the need for daily ultrasound examinations. The sensitivity of each estrus detection method was defined as the number of times an alert was given by a system during the REP (true positive, TP) divided by the total number of REP (true estrus). The PPV was the number of TP compared with the total number of alerts for that method.

Each cow was fitted with a commercial activity meter (ActoFIT, version 2015, DeLaval) on the neckband. Increased activity was monitored via the algorithm included in the DelPro Farm Manager software (DeLaval). This algorithm generated activity alerts at 3 possible levels $(+,++,+++)$, depending on the actual restlessness of the cow compared with her normal behavior. Often,,+++ , and +++ alerts were generated within the same estrus period. In this case, only the highest level of activity within the REP was considered. When only a + or ++ alert was generated for an estrus event, the timing of these was recorded. Each alert not associated with a REP was considered a false alarm (false positive, FP). Progesterone-based alerts were generated with a recently developed on-line monitoring system, P4 Monitoring Algorithm using Synergistic Control (PMASC), which consists of a mathematical model describing the different parts of the $\mathrm{P} 4$ profile (Adriaens et al., 2017) and a statistical process control chart to indicate luteolysis (Adriaens et al., 2018). An alert was identified as TP if followed by a REP within $24 \mathrm{~h}$; all other alerts were considered FP. The sensitivity and PPV of the different estrus detection systems were determined for a period of $41 \mathrm{~d}$ (i.e., periods $1+2$ ), allowing for a training period of $12 \mathrm{~d}$ for PMASC, during which only $\mathrm{P} 4$ samples were taken.

The preovulatory LH surge was monitored as a proxy for ovulation (period 2) between d 28 and 53 of the trial. If the presence of a preovulatory follicle was confirmed after a decline in milk $\mathrm{P} 4$ concentration, blood samples were collected in serum Vacutainer tubes (Becton, Dickinson and Co., Franklin Lakes, NJ) every $2 \mathrm{~h}$ from the jugular vein with a disposable 20-gauge needle (Becton, Dickinson and Co.) for a period of 72 $\mathrm{h}$, starting $36 \mathrm{~h}$ after the moment when raw milk $\mathrm{P} 4$ started a consistent decline toward concentrations $<5$ $\mathrm{ng} / \mathrm{mL}$. A preliminary study showed that this number of blood samples (36 samples) was needed to capture all LH surges. If a cow exhibited metestrus bleeding, sampling was stopped. The clotted blood samples were centrifuged at 2,300 $\times g$ to collect the serum, from which 3 aliquots were stored at $-20^{\circ} \mathrm{C}$. At the end of the trial, the serum LH concentrations were measured on a BEP2000 system (Siemens Healthcare Diagnostics, Marburg, Germany) with a commercially available bovine LH ELISA kit (Abnova, Taipei City, Taiwan), having intra-assay coefficients of variation of $6.3 \%$ (average LH: $13.2 \mathrm{ng} / \mathrm{mL}$ ) and 5.5\% (average LH: $45.2 \mathrm{ng} / \mathrm{mL}$ ) and a limit of quantification of $3 \mathrm{ng} / \mathrm{mL}$. All samples of one REP were analyzed on the same ELISA plate. The presence of an LH surge was visually determined by plotting time against $\mathrm{LH}$ concentration and the moment of the LH surge was identified as the maximal LH concentration, which was between 17 and $49 \mathrm{ng} /$ $\mathrm{mL}$. In all cases, the surge concentration was more than 8-fold greater than the baseline LH concentration. In total, LH samples were collected during 24 REP from 22 cows over $25 \mathrm{~d}$. To obtain unbiased results, cows with severe health or fertility problems known to affect endocrinology (Dobson et al., 2008; Walker et al., 2008) were excluded from this part of the study. These included 1 cow treated for milk fever, 1 cow that was severely lame, 1 cow with endometritis, 3 cows treated for a luteal cyst, and 3 cows with a follicular cyst or that became anestrous after a normal cycle. A total of 15 REP (9 primiparous, 6 multiparous cows, $158 \pm 53$ DIM, BCS $3.2 \pm 0.3$; mean $\pm \mathrm{SD}$ ) were retained for the final analysis.

The time interval (TI) between each TP activity or P4 alert and the moment of maximal LH concentration was calculated in hours, and are referred to hereafter as $\mathbf{T I}_{\mathbf{A C T}}$ and $\mathbf{T} \mathbf{I}_{\mathbf{P M A S C}}$, respectively. Additionally, we evaluated the relationships between the LH surge and several model-based indicators derived from PMASC. For this purpose, we calculated a TI with (1) the inflection point of the decreasing Gompertz function describing the luteolysis $\left(\mathbf{T I}_{\mathbf{I P}}\right)$; (2) the intercept of the tangent line at the inflection point with the time-axis $\left(\mathbf{T I}_{\mathbf{I C}}\right)$; (3) the moment that the model surpassed a fixed threshold of $3,5,7$, or $10 \mathrm{ng} / \mathrm{mL}$ ( $\mathbf{T I}_{\text {TMOD_x }}$ ); and (4) the moment the model undercut 85, 90, or $95 \%$ of the maximum $\mathrm{P} 4$ model concentration minus the baseline $\left(\mathbf{T I}_{\mathbf{T B} \_\mathbf{X}}\right)$, with $\mathrm{X}$ representing the respective percentages and thresholds (Adriaens et al., 2017, 2018).

Thirty-five REP were detected in 22 cows during periods $1+2$. The number of alerts given per system, together with their sensitivity and PPV, are summarized 
Table 1. Summary of the alerts and sensitivity (Se), positive predictive value (PPV), and time intervals $\left(\mathrm{TI}_{\mathrm{X}}\right)$ for the different estrus detection systems

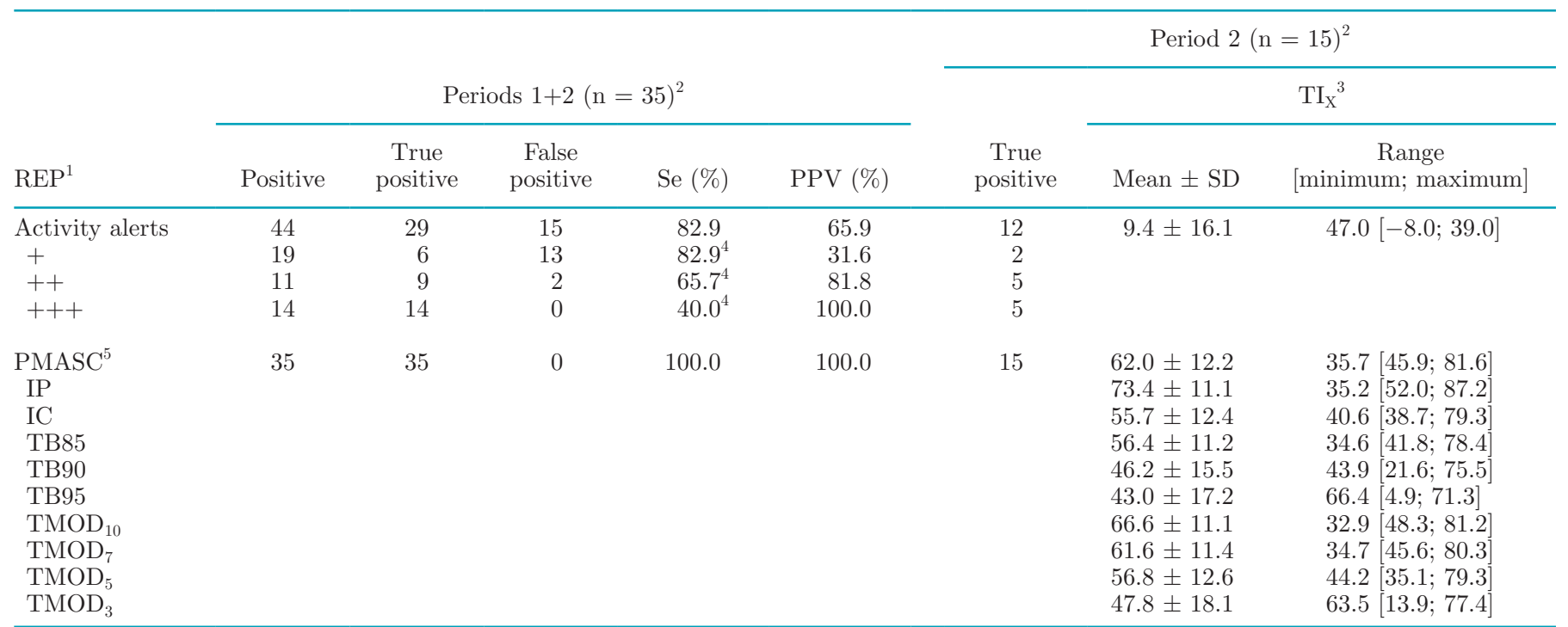

${ }^{1}$ Reference estrus period.

${ }^{2}$ Positives $=$ estrus alerts generated with progesterone (P4)-based monitoring algorithm using synergistic control (PMASC) or the activity-based system; true positives $=$ alerts associated with a REP; false positives $=$ alerts not associated with a REP.

${ }^{3} \mathrm{TI}_{\mathrm{X}}=$ time interval with alert or model-based indicator and the preovulatory LH surge.

${ }^{4}$ The sensitivity for the activity alerts was calculated cumulatively, using all true positives $(+/++/+++)$ for "+", only ++ and +++ alerts for "++" and only +++ alerts for "+++".

${ }^{5}$ PMASC model-based indicators: (1) time intervals with inflection point of the decreasing Gompertz function describing the luteolysis ( $\mathrm{TI}_{\mathrm{IP}}$ ); (2) intercept of the tangent line at the inflection point with the time axis $\left(\mathrm{TI}_{\mathrm{IC}}\right) ;(3)$ the moment the model undercuts 85,90, or $95 \%$ of the maximum P4 model concentration minus the baseline $\left(\mathrm{TI}_{\mathrm{TB} \_\mathrm{X}}\right)$, with $\mathrm{X}$ representing the respective percentages; and (4) the moment that the model surpassed a fixed threshold of $3,5,7$, or $10 \mathrm{ng} / \mathrm{mL}\left(\mathrm{TI}_{\mathrm{TMOD} \_\mathrm{X}}\right.$ ), with X representing the respective thresholds (Adriaens et al., 2017, 2018).

in Table 1 . The activity alerts are expressed according to their maximum activity level $(+,++$, or +++$)$ for each REP. When all 3 activity levels were considered, a sensitivity of almost $83 \%$ was obtained. However, estrus detection solely based on the "+" level of activity was not reliable, because 13 of the 19 "+" alerts $(68.4 \%)$ were false alarms (PPV of 31.6\%). These moderate increases in activity are most likely to be caused by dominance fighting or environment-related factors. Using only the ++ and +++ alerts decreased the number of false alarms to 8.0 and $0.0 \%$, respectively, but also reduced the sensitivity to 65.7 and $40.0 \%$. The main advantage of activity meters over traditional visual estrus detection is the automated and continuous nature of the system, which reduces the farmer's time required for estrus detection. The sensitivity and PPV in this study, however, did not meet the standards of a minimal sensitivity of $80 \%$ and a minimal specificity of 99\% (ISO 20966:2007) required for sensor systems. Moreover, the current results included only cows that were verified to be cycling and healthy at the start of the trial. Accordingly, the FP rate for ++ and +++ alerts might increase further when including cows with disorders (e.g., nymphomaniac animals with follicular cysts). Additionally, activity scoring does not identify true silent ovulations (17.1\% in our study).

The PMASC system identified all 35 REP in the 41-d study period and did not generate any false alerts (sensitivity and PPV of 100\%). In a large study conducted by Friggens et al. (2008), comparable sensitivities of 93.3 to $99.2 \%$ were reached, using the model described by Friggens and Chagunda (2005). This result might be influenced by the way a REP was defined. The additional exams of the ovaries using ultrasonography confirmed each estrus, and physiology dictates that estrus cannot occur during luteal phases of the cycle. Therefore, the current method applied is a sound one, and no better alternative is available today.

Typically, health affects the intensity of estrus, which influences the performance of activity-based systems. As P4-based systems are limited to the identification of luteolysis, for which the link with the quality of the follicle or oocyte is unclear, the value of these P4-based systems might be overestimated. Improved practice-based insight on factors that affect the chance of successful conception (e.g., BCS, disease, insufficient uterus tone) is therefore needed before the real value of identifying each luteolysis can be estimated. To avoid 
potential confounding effects of health status, the second part of this study, which investigated the link between the LH surge and the respective alerts generated, used only REP from healthy cows (period 2, Table 1).

The concentrations of baseline serum $\mathrm{LH}$ and that of the LH surge were, respectively, $1.46 \pm 0.84$ and $36.3 \pm$ $11.3 \mathrm{ng} / \mathrm{mL}$ (mean $\pm \mathrm{SD}$ ), and approximately 4 measurements were involved per peak (i.e., the LH surge developed and diminished within $8 \mathrm{~h}$ ). An overview of LH data and P4 profiles centered around the peak of the LH surge is illustrated in Figure 1. The TI between the LH surge and the different alerts $\left(\mathrm{TI}_{\mathrm{ACT}}, \mathrm{TI}_{\mathrm{PMASC}}\right.$, $\left.\mathrm{TI}_{\mathrm{IC}}, \mathrm{TI}_{\mathrm{IP}}, \mathrm{TI}_{\mathrm{MOD} \_\mathrm{x}}, \mathrm{TI}_{\mathrm{TB} \_\mathrm{x}}\right)$ is summarized in Table 1 . Three of 15 REP were not associated with any activity alert. Of the others, 2 had only a "+" alert, which was shown previously to be unreliable for estrus detection. The range in time from alert to LH surge varied from $39 \mathrm{~h}$ before the LH surge to $8 \mathrm{~h}$ after it (SD of $16.1 \mathrm{~h}$ ). Roelofs et al. (2005) reported that ovulation occurred $29.3 \pm 3.9 \mathrm{~h}$ after the onset of an increased number of steps (TI between 22 and $39 \mathrm{~h}$ ) and $19.4 \pm 4.4 \mathrm{~h}$ after the end of the increased number of steps (TI between 12 and $35 \mathrm{~h}$ ), which suggests that alternative sensor systems and data-processing algorithms might improve our results. However, evaluating these was outside the scope of this study.

The average TI from luteolysis detected with the PMASC system to the LH surge was $62 \mathrm{~h}$, with a minimum of 46 and a maximum of $82 \mathrm{~h}$, resulting in a range of $36 \mathrm{~h}$ (SD of $12.2 \mathrm{~h}$ ). The IP, as well as TB85, $\mathrm{TMOD}_{10}$, and $\mathrm{TMOD}_{7}$ performed similarly (range $\mathrm{TI}_{\mathrm{X}}$ of 33-35 h, SD: 11.1 to $11.4 \mathrm{~h}$ ). With a luteal-phase milk $\mathrm{P} 4$ concentration between 20 and $30 \mathrm{ng} / \mathrm{mL}$ and a follicular-phase milk $\mathrm{P} 4$ concentration of approximately $2.5 \mathrm{ng} / \mathrm{mL}$, TB85 represents the moment that the model first entered the range between $5.1[2.5+$ $0.15 \times(20-2.5)]$ and $6.6 \mathrm{ng} / \mathrm{mL}[2.5+0.15 \times(30$ - 2.5)]. Using the TB85 indicator, and thereby taking into account the decline in milk $\mathrm{P} 4$ compared with the absolute maximal milk $\mathrm{P} 4$ concentration, resulted in a lower TI range than when a fixed threshold of $5 \mathrm{ng} /$ $\mathrm{mL}$ was used in the model $\left(\mathrm{TI}_{\mathrm{TMOD} 5}\right)$. When a fixed threshold of $10 \mathrm{ng} / \mathrm{mL}$ was used $\left(\mathrm{TI}_{\mathrm{TMOD} 10}\right)$, however, an even smaller range of $33 \mathrm{~h}$ was noted (SD: $11.1 \mathrm{~h}$ ). These results demonstrate that the start of luteolysis might be more indicative of the timing of the LH surge than the time that milk P4 falls below the $5 \mathrm{ng} / \mathrm{mL}$ threshold. In the current study, samples were collected at each milking, which may not be economically viable. When sample collection frequency is lower, however, the model-based indicators would likely outperform the luteolysis alerts of PMASC. Therefore, using model-dependent rather than data-dependent guidelines might make the monitoring system more robust. Furthermore, these model-based indicators can be calculated for each profile, allowing flexibility to account for differences in shapes and absolute levels of the different estrous cycles, and thereby facilitating further optimization of insemination advice; for example, by fixing the slopedetermining parameters to physiologically relevant ranges when fewer samples are available (Meier et al., 2009).

The limited number of estrus events and the fact that only one farm was included in the study prevents us from identifying the optimal insemination time for alerts generated with these systems, and our observa-

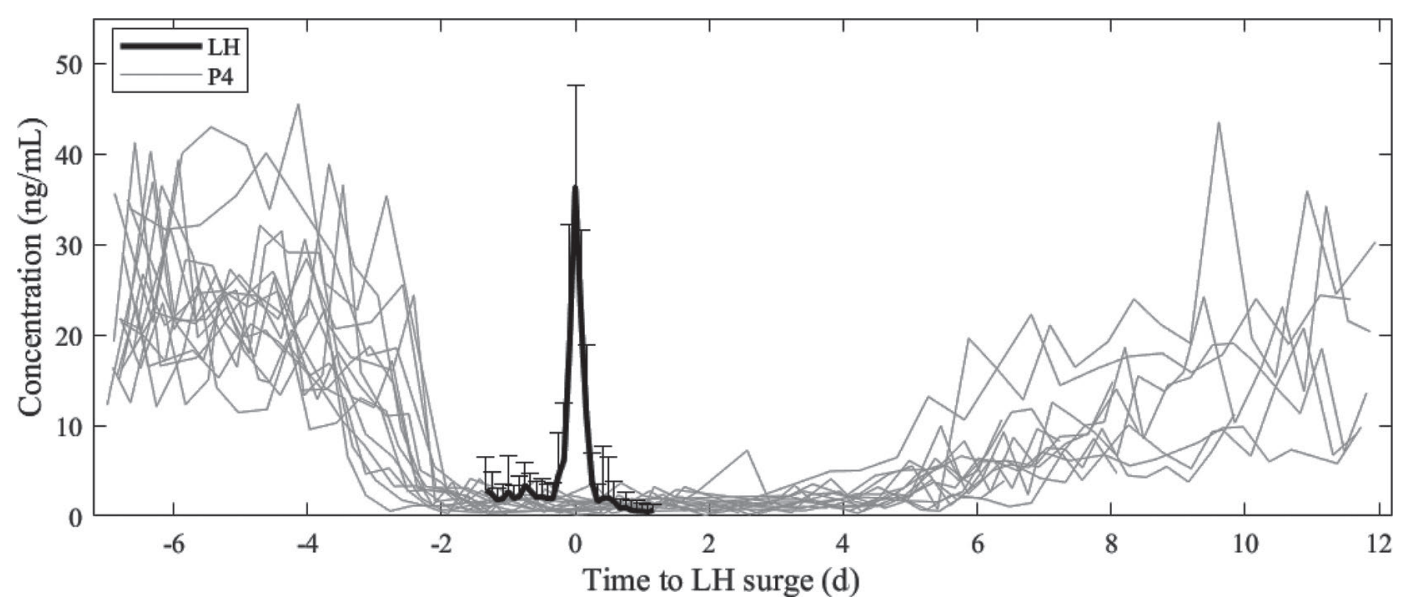

Figure 1. Overview of the progesterone (P4) profiles centered around the LH surge. A large variability in the P4 profiles is noted both before and after the LH surge, but all cows had an active corpus luteum, reflected in a P4 concentration $>5 \mathrm{ng} / \mathrm{mL}$ within $7 \mathrm{~d}$ after the LH surge. The error bars represent the SD to the mean over the 15 included LH surges. 
tions and results cannot be extended to genetically different herds. Therefore, more data should be collected to confirm or contradict our results, taking into account various environmental factors (e.g., physiology, genetics, or management). Unfortunately, studies that record ovulation time are time consuming, labor intensive, and expensive. Thus, smaller studies such as this one are valuable when they include a reference for ovulation while accounting for more than one estrus detection system. However, we confirm the need for additional research including different estrus detection tools and a good reference for the time of ovulation. We suggest that frequent blood sampling to monitor the LH surge is a useful alternative to frequent transrectal ultrasound to monitor disappearance of the ovulatory follicle.

To conclude, we found that P4 monitoring allowed for accurate identification of luteolysis and outperformed activity-based estrus detection system in terms of sensitivity and PPV. Moreover, using LH as a proxy for ovulation, the P4-based system and derived model indicators can provide a more reliable identification of the correct time for insemination.

\section{ACKNOWLEDGMENTS}

This work was supported by the Institute for the Promotion of Innovation through Science and Technology in Flanders, Belgium (IWT; IWT-LA project 110770). Ines Adriaens and Ben Aernouts are supported by the Fund for Scientific Research (FWO) Flanders (grant numbers 11ZG916N and 12K3916N, respectively). We thank Shahbaz Bashir (Division of Animal and Human Health Engineering, Leuven, Belgium) and Mathilde Tinel (Izegem, Belgium) for their assistance during the blood sampling. Lattec (Hillerød, Denmark) supported this study by supplying additional P4 sticks and modifying the Herd Navigator software to take samples at every milking event.

\section{REFERENCES}

Adriaens, I., T. Huybrechts, K. Geerinckx, D. Daems, J. Lammertyn, B. De Ketelaere, W. Saeys, and B. Aernouts. 2017. Mathematical characterization of the milk progesterone profile as a leg up to individualised monitoring of reproduction status in dairy cows. Theriogenology 103:44-51. https://doi.org/10.1016/j.theriogenology .2017.07.040.

Adriaens, I., W. Saeys, T. Huybrechts, C. Lamberigts, K. Geerinckx, J. Leroy, B. De Ketelaere, and B. Aernouts. 2018. A novel system for online fertility monitoring based on milk progesterone. J. Dairy Sci. 101:8369-8382. https://doi.org/10.3168/jds.2017-13827.

Dobson, H., S. Walker, M. Morris, J. Routly, and R. Smith. 2008. Why is it getting more difficult to successfully artificially inseminate dairy cows? Animal 2:1104-1111. https://doi.org/10.1017/ S175173110800236X.

Friggens, N. C., M. Bjerring, C. Ridder, S. Højsgaard, and T. Larsen. 2008. Improved detection of reproductive status in dairy cows using milk progesterone measurements. Reprod. Domest. Anim. 43:113-121.

Friggens, N. C., and M. G. G. Chagunda. 2005. Prediction of the reproductive status of cattle on the basis of milk progesterone measures: Model description. Theriogenology 64:155-190. https:// doi.org/10.1016/j.theriogenology.2004.11.014.

Hockey, C. D., J. Morton, S. Norman, and M. McGowan. 2010a. Improved prediction of ovulation time may increase pregnancy rates to artificial insemination in lactating dairy cattle. Reprod. Domest. Anim. 45:e239-e248. https://doi.org/10.1111/j.1439-0531 2009.01548.x.

Hockey, C. D., J. M. Morton, S. T. Norman, and M. R. McGowan. 2010b. Evaluation of a neck mounted 2-hourly activity meter system for detecting cows about to ovulate in two paddock-based Australian dairy herds. Reprod. Domest. Anim. 45:e107-e117. https://doi.org/10.1111/j.1439-0531.2009.01531.x.

ICAR (International Committee for Animal Recording). 2014. ICAR Recording Guidelines. Accessed Sep. 6, 2015. https://www.icar .org/index.php/icar-recording-guidelines/.

Meier, S., J. Roche, E. Kolver, G. Verkerk, and R. Boston. 2009. Comparing subpopulations of plasma progesterone using cluster analyses. J. Dairy Sci. 92:1460-1468. https://doi.org/10.3168/jds.2008 -1464 .

Roelofs, J. B. 2005. When to inseminate the cow? Insemination, ovulation and fertilization in dairy cattle. $\mathrm{PhD}$ Thesis. Wageningen Institute of Animal Sciences, Wageningen, the Netherlands.

Roelofs, J. B., F. J. C. M. Van Eerdenburg, N. M. Soede, and B. Kemp. 2005. Pedometer readings for estrous detection and as predictor for time of ovulation in dairy cattle. Theriogenology 64:1690-1703. https://doi.org/10.1016/j.theriogenology.2005.04.004.

Walker, S. L., R. F. Smith, D. N. Jones, J. E. Routly, and H. Dobson. 2008. Chronic stress, hormone profiles and estrus intensity in dairy cattle. Horm. Behav. 53:493-501. 